\title{
MODALIDADES DE REALIMENTACIÓN EN LA ASIGNACIÓN DE ACORDES A UNA MELODÍA Y EN EL DISEÑO DE LA PROGRESIÓN DE FUNCIONES ARMÓNICAS
}

\author{
Horacio Alberto Lapidus*
}

\begin{abstract}
Paper that reports on research developed -as a magister thesis on the topic of feedback in musical education software, specifically directed to the learning of certain aspects of harmonization of tonal melodies in classical style. For its analysis, an intelligent tutor type application was implemented, and tested with the purpose of obtaining data that allowed to compare the use of two types of feedback. Two sample groups were made with students from the Music Department of Universidad Nacional de Colombia in Bogotá. The study suggests the convenience of combining both types of feedback, so that the user can migrate from one modality to another at will, under certain conditions.
\end{abstract}

\section{RESUMEN}

Informe sobre la investigación desarrollada - como tesis de maestría ${ }^{1}$ — en torno a la realimentación en el software de educación musical, específicamente enfocada ciertos aspectos del aprendizaje de la armonización de melodías tonales en estilo clásico. Para su análisis, se implementó una aplicación de tipo tutor inteligente, que fue puesta en experimentación con el fin de obtener datos que permitieron comparar la aplicación de dos tipos de feedback en la utilización de dicho software. Se conformaron dos grupos de muestra con estudiantes del Departamento de Música de la Universidad Nacional de Colombia, en Bogotá. El estudio sugiere la conveniencia de combinar los dos tipos de feedback comparados, de modo que el estudiante pueda migrar voluntariamente de uno al otro, bajo ciertas condiciones.

Palabras clave: Armonización de melodías. Tutores inteligentes para educación musical. Feedback en software educacional. Análisis armónico.

\section{Introducción}

El interés de muchos profesores por la innovación tecnológica, en ciertas ocasiones afortunadas, es lo suficientemente amplío como para no reducirse al simple deseo de enriquecer los medios disponibles (piano, tablero pentagramado, grabaciones, partituras), sino que se acompaña de un cuidado por el ejercicio de una pedagogía respetuosa de la inteligencia del estudiante, lo cual es decir bastante en el marco de una tradición habitualmente repetitiva y memorística de la enseñanza musical. Estos maestros tienden a descubrir, entonces, que el atractivo técnico o de diseño de muchos programas educativos no disimula el conformismo didáctico de su propuesta. Memoria, repetición, tests, conforman un paradigma difícil de contrarrestar. Esto parece concordar con el rápido desencanto que suele producirse entre muchos estudiantes enfrentados al uso de dicho material. En efecto, el software musical educativo orientado al lenguaje musical, tra-

\footnotetext{
*Profesor Asociado Universidad Nacional de Colombia - Facultad de Artes. Sede Bogotá.

${ }^{1}$ Maestría en Tecnologías de la información aplicadas a la educación, Universidad Pedagógica Nacional, Bogota, Colombia, 2001. Director. Profesor Luis Facundo Maldonado Granados.
} 
dicionalmente estuvo basado en un tipo de aprendizaje conducente a la creación de asociaciones firmes entre elementos conceptuales, auditivos y gráficos.

Algunos campos de la formación básica musical, y en particular la armonía, ya han sido objeto de numerosos aportes en diversas aplicaciones, por lo general destinadas al entrenamiento de habilidades básicas, tales como el reconocimiento de acordes sonoros o escritos, o la identificación de formas cadenciales. El estado del arte nos muestra que sólo unas pocas de estas aplicaciones incluyen, hasta el momento, recursos de feedback con un alcance que supere la simple valoración de la respuesta como correcta/incorrecta, o del análisis elemental de errores de audición o de concepto. Los nuevos tipos de feedback adaptativo responden a enfoques cuyo interés se centra en el proceso cognitivo de cada estudiante al interactuar con el material propuesto.

En nuestro caso, la propuesta pedagógica implementada pretende poner en práctica una idea de contextualización, según la cual las actividades de aprendizaje de la música deberían estar enmarcadas, preferiblemente, en la simulación o la práctica efectiva de situaciones reales, de modo que los ejercicios destinados a la incorporación de habilidades específicas no se separen de un entorno musical capaz de proporcionarles significado e interés. En este sentido, se habilitó la aplicación de software desarrollada, para soportar un tipo de melodías más próximas a la música real que los tradicionales cantos dados de los ejercicios elementales, pudiendo estar ornamentadas. Por otra parte, se plantea a los usuarios resolver el problema de armonización de toda una melodía, en vez de producir o reconocer algunos encadenamientos aislados de acordes como tienden a proponer otras aplicaciones educativas.

Pero el aspecto más específico de este estudio radica en la contrastación dedos modalidades de feedback adaptativo: una de ellas más "automática", basada en reacciones espontáneas del software al detectar problemas de estilo (o trasgresión de reglas prácticas) por parte del estudiante, y un tipo de feedback "por solicitud", ofrecido cuando el usuario desea evaluación de cualquiera de los estados parciales (acordes nuevos) en la solución del problema.

\section{Algunos antecedentes}

En las últimas tres décadas se han desarrollado sistemas musicales expertos, capaces de realizar tareas tan complejas como componer sonatas en la tradición clásica, 0 aprender (mediante redes neuronales) características particulares de uno u otro estilo. La bibliografía consultada demuestra que, a nivel educativo, ciertos desarrollos interesantes parecen centrarse en el tipo de feedback ofrecido como el output principal del dispositivo de enseñanza-aprendizaje.

En el campo de educación musical, los programas de instrucción asistida por computador (CAl) provistos de algún grado de inteligencia artificial, han ido superando pese a las dificultades de su dispendioso desarrollo- a las aplicaciones iniciales de ejercitación y práctica, ya que al acercarse a modelos de docencia humana, logran identificar características del estudiante y sus respuestas, lo que les permite ubicar y aplicar estrategias de enseñanza específicas. Sin embargo, en un panorama bastante abarcador, Berz y Bowman reportan todavía una carencia de modelos satisfactorios para interpretar estas dinámicas relaciones entre usuario y este tipo de entornos pedagógicos (Berz y Bowman, 1994). 
Por su parte, en un interesante informe, John W. Schaffer (Schaffer, J.W., 1988), aplica la fundamentación pedagógica de los tutores inteligentes al análisis del Harmony Coach, un programa que él realizó, capaz de evaluar la conducción de voces en armonizaciones de corales -estilo J.S.Bach--, el cual constituye un antecedente muy cercano del software desarrollado para esta investigación. El autor explica que los avances en inteligencia artificial, permiten explorar mejor la forma en que las personas realizan su adquisición y utilización del conocimiento. Al recordar que los sistemas expertos acumulan y emplean un conocimiento similar al de los seres humanos, enumera y describe las posibilidades de diseño de estos sistemas, basados unos en lógicas proposicionales y otros en lógicas predicativas. En ese sentido, su Harmony Coach se presenta como un tutor inteligente destinado a entrenar tareas específicas musicales de escritura de progresiones armónicas tonales, ayudando al estudiante en la creación de una secuencia armónica aceptable basada en una línea de bajo no cifrada o en una melodía².

Los reportes escritos sobre el uso del Harmony Coach no profundizan en modalidades alternativas de feedback. Por otra parte, un programa de armonización desarrollado a mediados de los 80 , MacVoice, era básicamente descriptivo de problemas de conducción de voces y de recomendaciones procedimentales, sin profundizar en la naturaleza de cada caso específico señalado como error.

En la actualidad, existen en el mercado varias aplicaciones de educación musical que incluyen el entrenamiento de algunas habilidades del campo armónico, como el reconocimiento auditivo de acordes individuales o de secuencias típicas en las que se suelen presentar. El feedback en dichas aplicaciones suele circunscribirse al tipo "respuesta correcta/incorrecta", y a veces orientan acerca del tipo de error.

Por fuera del plano evaluativo, todo el software musical proporciona feedback auditivo, y en ciertos casos análisis y ayudas automáticas para la realización musical (arreglos) del material armonizado por el usuario.

\section{Estudios sobre feedbacken instrucción programada}

\subsection{Evolución}

En la revisión histórica de la evolución del feedback en instrucción prog ramada que realizan Raymond Kulhavy y Walter Wager (Kulhavy y Wager, 1993), encuentran que el papel del feedback, en procesos de aprendizaje, fue visto como:

- Incentivo para mejorar el desempeño.

- Reacción "aprobatoria" capaz de conectar automáticamente la respuesta con el estímulo precedente.

- Información utilizable para validar o cambiar la respuesta dada.

Especialmente la segunda modalidad supone un enfoque conductista, orientado a un feedback tipo respuesta correcta/incorrecta, que estimuló la producción de una gran cantidad de material educativo.

Pero a partir de los años 70 se comienzan a generar aproximaciones alternativas, apoyadas en la falta de efectos sistemáticos del feedback-estímulo, demostrada en varios

${ }^{2}$ Schaffer,J.W.. W op. cit., p 1. 
estudios. Esto llevó a repensar los criterios sobre el tipo de información que debía proporcionarse al usuario tras sus respuestas, especialmente las erróneas.

Kulhavy y Wager anotan que el feedback demostró ser significativo en el aprendizaje cuando se provee después de la respuesta, y que lograba mayor eficacia si se dirigía a corregir errores, más que al aparecer tras una respuesta correcta. El artículo mencionado cita unos trabajos tempranos (Crowder N., 1960, 1961) en los cuales ya se contrarrestaba el concepto de los errores como pérdida, proponiendo que podían corregirse presentando información y no sólo dando al usuario la respuesta correcta.

\subsection{Feedback adaptativo}

Una definición amplia de feedback (Wager, W. y Wager, 8., 1986) ha sido expresa como .. "cualquier mensaje o pantalla que presenta el computador al alumno después de una respuesta" (o acción - input). Su propósito: modelar las percepciones del aprendiz. Esta posición se acerca al pensamiento actual de la psicología cognitiva y teorías de la información. No se restringe el feedbackal área de práctica, lo cual incrementa las oportunidades de instrucción. En general, conviene considerar el feedback como un input periódico para el aprendiz, que también cumple roles de soporte procedimental, motivación, etc. Es decir, sus funciones aparecen expandidas en la instrucción tecnológicamente asistida. A medida que la tecnología evoluciona, se van haciendo posibles nuevos tipos de feedback: desde simples textos, luego beeps, melodías-señal, ...hasta alcanzar hoy, la combinación de medios como información visual (texto, gráficas, animación, imagen digital, video), auditiva (sonidos generados por el computador, audio digital, MIDI, voz digitalizada), táctil, etc.

Sin embargo, Wager y Wager encuentran que aparentemente, los diseñadores de software tienen poco en cuenta el papel del feedback, pues una mayoría de productos realizan evaluaciones breves tipo "correcto/incorrecto", o apenas la actualización de los puntajes obtenidos. Es decir, no se consideran diferencias individuales entre los usuarios. Los diseñadores parecen apuntar a un desarrollo fácil y económico de las subrutinas, las que además deberán emplearse repetidamente para todas las acciones del usuario. La excepción sería el software desarrollado con componentes de feedback adaptativo.

Para implementar esta capacidad, deben considerarse entre otros, factores como: características del alumno, contenido de la instrucción, resultados esperados del aprendizaje, y el ambiente en que transcurrirá la práctica.

Gregory Sales (Sales, 1993), destaca la importancia de las estrategias adaptativas del feedback, y también las empleadas por los usuarios para obtenerlo. Entre ellas, un ejemplo mencionado es la posibilidad de que los estudiantes, en algún aspecto que puedan controlar, tengan la oportunidad de seleccionar el tipo de feedback.

En un estudio de Steven Ross y Gary Morrison (Ross, 8. y Morrison, G., 1993), se analizan y comparan distintas estrategias de feedback para programas de instrucción basada en computadores. En primer lugar ellos consideran las de respuesta correcta y conocimiento de la respuesta correcta, y luego una que nos interesa en especial: el feedback que permite responder hasta acertar (“AUC: answer-until-correct”), ventajosa porque varía el grado de contacto del estudiante con los contenidos, en la medida del número de intentos que necesita para escoger una respuesta adecuada. 
Avanzando sobre la calidad de la adaptación, aparece el concepto de feedback elaborativo. kulhavy y Stock (1989) describen dos estadios en el feedback: verificación, la simple información correcto/ incorrecto, y la elaboración, que consiste en proporcionar información posterior a dicha comprobación.

Pero en instrucción computarizada (CAI), la adaptación no se reduce a presentar unidades de información de acuerdo a la respuesta elegida, sino que supone cierto análisis de dichas respuestas (cada una y en forma global), respecto del cual el feedback estará optimizado en función del aprendizaje de un punto específico. En este nivel, tendremos los tutores inteligentes, generados con la intención de sustituir algunas funciones del instructor humano, y cuyo desarrollo dispendioso hizo que los principales modelos fueran, hasta hace unos años, básicamente demostrativos.

Ross y Morrison presentan otras orientaciones, efectivas y menos complejas: en particular nos interesa el análisis sensible a la respuesta. Tales estrategias suponen un enfoque más cognitivo, que al identificar el dominio de los errores cometidos, permiten inferir cuáles reglas presentan dificultades, proporcionando un tipo especifico de información correctiva necesaria para resolver el problema planteado. Esta perspectiva cognitiva permite examinar el papel de la personalización y la adaptación del feedbacken los programas de instrucción asistida por cornputador.

\section{Modelo de la situación de enseñanza/aprendizaje implementada}

\subsection{Requerimientos cognitivos de la respuesta}

En el problema de armonización de una melodía empleado para este estudio, cada acorde nuevo que introduce el usuario es un paso de la respuesta, o la solución a un subproblema. En la colocación de este acorde están involucrados los siguientes niveles cognitivos:

\subsubsection{Nivel motor}

Ejecución del acorde. Implica controlar manos y dedos, las teclas a pulsar, la duración de las notas tocadas, la intensidad (velocidad de bajada de las teclas).

Muchas veces las cadenas motoras se inician pulsando las flechas de cursor (izquierda-derecha) para ubicar la posición del nuevo acorde. La inclusión automatizada de este primer paso se logra tras varios intentos de ensayo/error.

\section{1.2. Nivel de discriminaciones múltiples}

Este aspecto de la respuesta permite diferenciar, para una función armónica, entre sus posibles estructuras (tres o cuatro notas), y sus diversas posiciones (estado fundamental o inversiones). Utilizar una u otra forma tiene condiciones de uso a nivel de la progresión de acordes, y presenta diferencias auditivas que el usuario está en capacidad de comprobar para efectuar su selección de acuerdo - dentro de ciertos límites- a sus preferencias. Se trata, entonces, del empleo y desarrollo de capacidades de discriminación auditiva. 


\subsubsection{Nivel conceptual}

Al analizar el sector de la melodía al que el sujeto asignará un acorde; debe decidir qué notas de dicho sector quedarán incluidas en el acorde, es decir, podrán considerarse como pertenecientes a él. El concepto empleado aquí es la estructura del acorde. Luego deberá establecer qué carácter tienen las notas descartadas (es decir, las que no gozan del atributo pertenece al acorde). Aquí juega una nueva subclasificación: su pertenencia o no a algunas de las clases de notas ornamentales (con conceptos específicos que definen los atributos de cada una).

\section{1.4. Nivel de aplicación de principios (proposiciones)}

\section{Ejemplos:}

- Si las notas extrañas no encajan en categorías ornamentales, el acorde no responde a parámetros de estilo (cambiarlo).

—El final de la melodía lleva algún encadenamiento de acordes típico; es decir, una cadencia, etc.

\subsubsection{Nivel de solución del problema}

En cada respuesta particular, se trata de lograr que el acorde puesto satisfaga simultáneamente las condiciones de estilo para:

- Estructura (construido por 3ras., tres o cuatro notas).

- Pertenencia tonal (debe estar entre los que pueden incluirse o relacionarse con la tonalidad de la pieza en un lenguaje clásico).

- Correspondencia acorde-melodía.

- Relaciones de progresión con el acorde anterior y posterior, y en el caso de tratarse de los acordes finales, encajar en el/los modelo(s) de cadencia preferibles.

\subsubsection{Totalidad de la armonización}

Además de satisfacer las "soluciones" particulares, el resultado final deberá garantizar algunas condiciones como:

- Ajuste a un modelo cadencial en el último grupo de acordes (varía entre 2 y 4 acordes).

- Presentar un porcentaje mayoritario de progresiones preferibles (aquéllas con relación de 5 . descendente entre las notas fundamentales de 2 acordes seguidos).

- Presentar un ritmo armónico coherente en sí mismo, con la cifra de compás y con la melodía, etc.

Evidentemente, aqui se combina la aplicación del conocimiento de varios principios, para lograr un balance adecuado que haga "satisfactoria" (ajustada a estilo) tanto la solución general como la utilización de cada acorde especifico.

\subsection{Factores intervinientes en la respuesta}

En el proceso de generación de una respuesta individual (cada acorde) intervienen, probablemente en una secuencia variable para cada individuo, y según sus diversos niveles de experiencia, una serie de aspectos entre todos los mencionados más arriba. 
Cuantos más de estos factores logren incorporarse a la decisión, tendremos la posibilidad de un aprendizaje más completo y un resultado cualitativamente superior.

En general, las categorías de esos factores intervinientes pueden agruparse en:

- Experiencia previa.

- Feedback puntual sobre una decisión tomada.

- Feedback general sobre el resultado total de la armonización.

- Autovaloración (conceptual y auditiva), del resultado de las respuestas.

\subsection{Derivación de un modelo pedagógico}

En Modelos didácticos (Escudero, 1981) se intenta definir un modelo de aprendizaje de carácter sistemático, abarcando la situación de enseñanza, el contexto educativo, factores institucionales y de contexto social. El autor establece cuatro características de un enfoque sistemático:

— Determinación precisa de niveles de entrada para los alumnos de un programa educativo.

— Establecimiento de objetivos (formulados en términos conductuales).

— Selección de estrategias, medios, recursos y materiales adecuados.

- Evaluación del proceso.

En esta perspectiva, Escudero propone profundizar la relación entre objetivos y proceso de aprendizaje, centrándose en el análisis de tareas, enmarcado en un modelo del proceso de enseñanza. De allí se deriva el siguiente esquema de un modelo específico de educación musical, referido a una situación de aprendizaje en ambiente computarizado, orientado al dominio de aspectos básicos en la armonización de melodías.

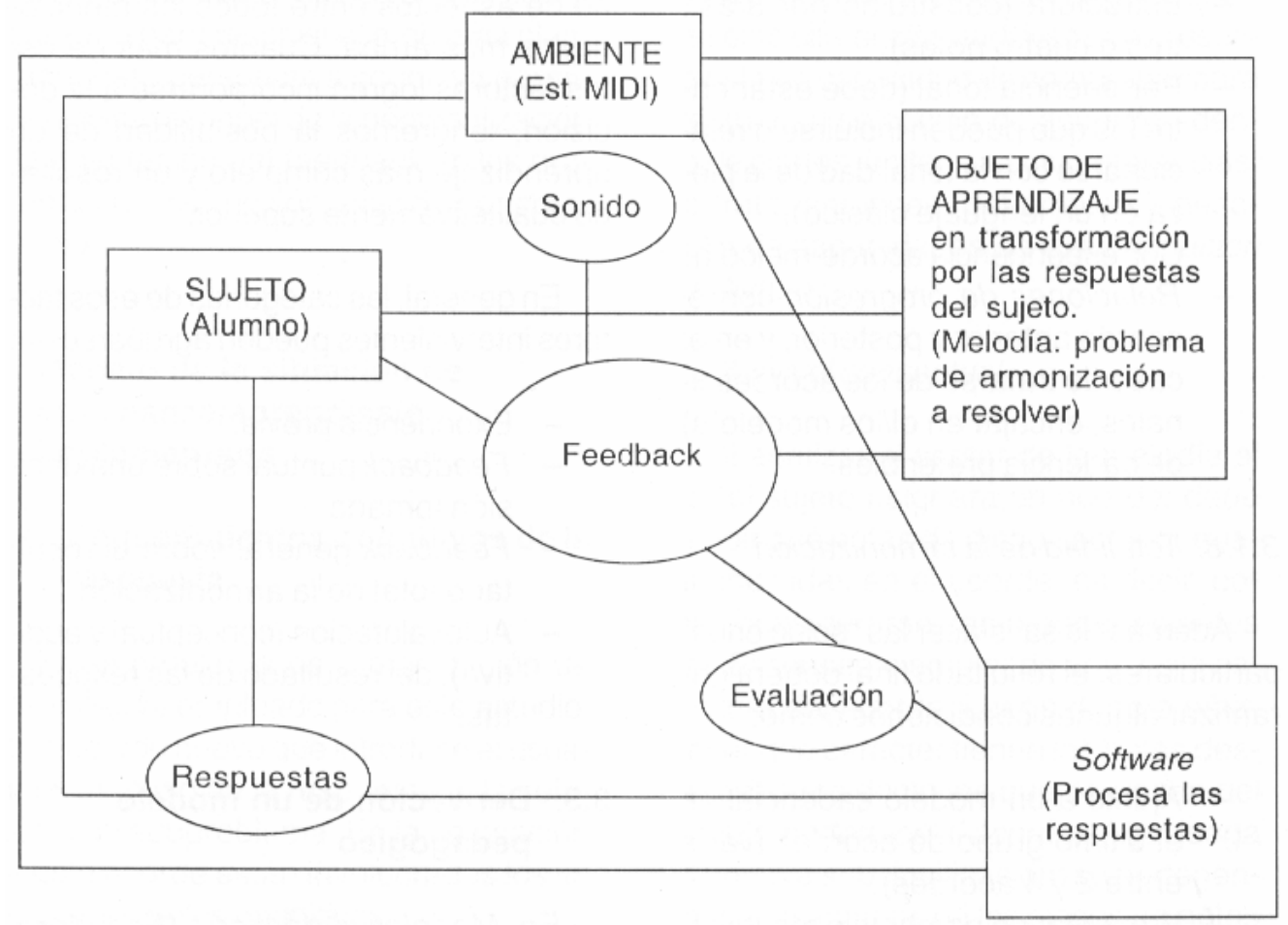

Figura 1. Esquema funcional del modelo pedagógico. 


\section{Análisis del feeback en el modelo}

La situación de enseñanza/aprendizaje implementada para el experimento supone diversos niveles en los que el programa reacciona (feedback) ante cada aspecto de las respuestas del usuario, parciales y totales.

- Al cargar la melodía, el estudiante puede solicitar un feedback auditivo, que asocia a la versión escrita en la medida en que su formación previa lo permita.

- Al realizar cada "movimiento" de su trabajo (colocación de los acordes), recibirá según el caso, diversos niveles de realimentación automática. En primer lugar, escuchará el acorde tocado en un teclado musical: asociación entre su concepto actual del acorde, y su significado sonoro/funcional.

- Si aparece un cuadro de diálogo aclarando los límites de tesitura, deberá reubicar su acorde dentro de ella. Esta opción puede asimilarse a un tipo de feedback que reacciona ante la respuesta incorrecta, pero con indicación del tipo de error. Se induce al alumno a discriminar entre las octavas del teclado que sí están habilitadas, y las que no.

- De un modo similar, el estudiante puede ser advertido de que su acorde no está registrado para la tonalidad de la pieza, lo cual, unido a su representación en la partitura, le permitirá confrontar el error con sus propios conceptos acerca de qué acordes corresponden a una tonalidad dada. Sin embargo, pese a identificar el error objetivo, el feedback no está diferenciando entre las posibles causas de tal error, que podrían orientar-se más a dificultades conceptuales, o bien motoras.

- Si la estructura y la tesitura del acorde tocado fueron aceptadas, el feedback consistirá en citrar(denominar) la función de dicho acorde respecto de la tonalidad indicada de la melodía. Esta realimentación está operando como información conceptual, en el sentido que se trata de una clasificación basada en el análisis de atributos del acorde tocado y de carácter unívoco (a tal acorde en tal tonalidad, un cifrado específico). El estudiante necesita la información provista por ese cifrado para abstraer las relaciones entre los sucesivos acordes.

- Una vez cifrado el acorde, el estudiante recibirá o podrá solicitar realimentación según sea el tipo de feedback asignado a su grupo- acerca de su viabilidad, la cual será brindada en tres planos:

a) Ornamentación resultante.

b) Problemas de correspondencia.

o) Problemas de progresión armónica.

a) En el primer caso se trata de una información analítica que le ayudará a comprender cómo interactúa con las notas de la melodía el acorde escogido. Esta es una información basada en conceptos de ornamentación, pero su manejo supone la aplicación de principios o reglas relacionales que permitan valorar la circunstancia específica de cada nota extraña al acorde. El estudiante podrá acceder-si lo desea- a definiciones y ejemplos escritos y sonoros de las categorías mencionadas, mediante navegación hipertextual.

b) Si el acorde puesto no satisface los requerimientos de a) (es decir, que todas las notas extrañas correspondan a categorías definidas) el usuario será informado de esta inconsistencia, con mención de cuáles notas impiden una adecuada correspondencia. Al 
seguir buscando acordes de reemplazo, el usuario intentará satisfacer ese requerimiento de correspondencia, hasta que en algún momento todas las notas extrañas de la melodía correspondan a categorías admitidas. Se emplea aquí también el criterio de intentarhasta-acedar, que es válido porque cada opción rechazada proporciona un análisis del error, que el alumno tiene posibilidad de aprovechar en beneficio de su comprensión del problema. Sin embargo, en determinado momento (después de 3 errores de correspondencia), el feedback pasará a un plano valorativo de la situación cognoscitiva del estudiante:

-Parece que usted no maneja bien el concepto de correspondencia acorde-melodía. Por favor consulte sobre este tema.

Aquí, el sujeto tiene la opción de proseguir con el mismo tipo de evaluación o cambiarla, lo cual le induciría a revisar su estrategia de solución, y su nivel conceptual respecto de un problema específico que ha detectado el programa.

o) De un modo similar al anterior, se señalan inconsistencias de estilo con respecto a los encadenamientos de acordes, considerados de a dos o de a tres para cada caso, más la confrontación de la solución con los modelos preferibles de cadencias para el final. Los señalamientos de error y sugerencias en este ámbito, permitirán acceder repetidamente a información más amplia, y el método intentar-hasta-acedar se enriquecerá mediante observaciones relacionadas con cada intento.

- Feedback do la solución general. Incluirá las respuestas parciales, y el porcentaje de progresiones preferibles, así como una "opinión" valorativa de ese porcentaje en términos de su sujeción al estilo estudiado, además de compararla con una posible versión anterior. Se indica, también, el valor numérico de los errores sin resolver. Queda abierta la opción de seguir corrigiendo, con lo cual tenemos un tipo de feedback adecuado al nivel de la solución del problema. Se incide en el nivel cognitivo del usuario, acercándole a una valoración de conjunto de dicha solución.

\section{Hipótesis: alternativas de feedback}

La cualidad interactiva de un ambiente instruccional computarizado supone la posibilidad constante de una "devolución" o respuesta del sistema a los pasos cumplidos por el estudiante. Esta realimentación puede asumir distintas modalidades, no todas excluyentes entre sí. En particular, se consideró pertinente comparar el comportamiento de los usuarios respecto de algunas de estas formas de feedback, especialmente si éstas representaban opciones pedagógicas distintas respecto del grado de autonomía con que el estudiante puede manejar su propio proceso de aprendizaje. Como hipótesis de trabajo, se asume un interés especial por uno de los dos tipos de feedback confrontados en la investigación: aquél cuyas evaluaciones puntuales son proporcionadas a solicitud del usuario, comparado con el feedback que espontáneamente interrumpirá su trabajo ante la detección de errores — también puntuales_de correspondencia, o progresión de acordes. Asumiendo que una conducción más autónoma del aprendizaje supone la posibilidad de decidir en qué momentos recurrir a esa realimentación evaluativa, se intentó validar esta opción, contrastando actitudes y resultados con la modalidad "automática" en la que el sistema proporciona feedback al producirse cada error. De esta manera, el interrogante principal, formulado a continuación, orientó el diseño del experimento. 


\subsection{Pregunta de investigación}

¿Existen diferencias significativas en el aprendizaje de la armonización de melodías realizado por estudiantes de música en un ambiente oomputarizado con feedback que interviene espontáneamente ante cada problema o error, y estudiantes de música que en condiciones similares, disponen de libertad para solicitar evaluación puntual de cualquiera de sus respuestas parciales?

La similitud de condiciones exigía, obviamente, un nivel semejante de conocimientos previos, iguales contenidos de información disponibles en el software, y los mismos problemas de armonización, que a efectos experimentales se plantearon como un proceso progresivo de tres trabajos.

\section{Representación del lenguaje musical}

El instrumento adecuado para cumplir las condiciones del experimento, debía ser capaz de presentar el problema mediante una interfaz convencional (sonido y notación musical en pentagrama), registrar la actividad del estudiante, modificar el estado de solución del problema a partir de sus acciones, evaluar estas modificaciones a la luz de una serie de reglas de conocimiento incorporadas, proporcionar información y realizar propuestas en función de dichas evaluaciones, apoyando la llegada del usuario a una solución adecuada, en cuyo proceso habrá adquirido conocimientos operantes. Ese objetivo coincide con los presupuestos de aquella forma evolucionada de software instruccional, que ya se ha mencionado como tutores inteligentes ( $\mathrm{TI})$.

La figura 2 muestra la pantalla de trabajo de la aplicación Acordes, desarrollada para los fines descritos.

Entre los esfuerzos de representación de conocimiento que se realizaron, cabe mencionar el "árbol de cadencias", durante cuyo recorrido el programa establece cuáles son las cadencias (encadenamiento de acordes conducentes a un final de frase) que podrá sugerir al usuario, en caso de que éste no las utilice, para cada melodía en particular.

El software admite varios tipos de usuarios, incluyendo administrador alumnos y usuarios independientes, y es capaz de registrar dos niveles de dificultad, ambos tipos de feedbacky datos resultantes de la actividad de cada usuario. También permite guardar, cargar y reabrir los trabajos realizados.

\subsection{Modelamiento del sistema tonal: las escalas}

West, Howelll y Cross (WEST et al., 85) exponen diversos intentos de modelamiento de la estructura musical que procuran ser independientes de las teorías musicales consideradas, en general, bastante interpretativas-vigentes históricamente para cada estilo. 


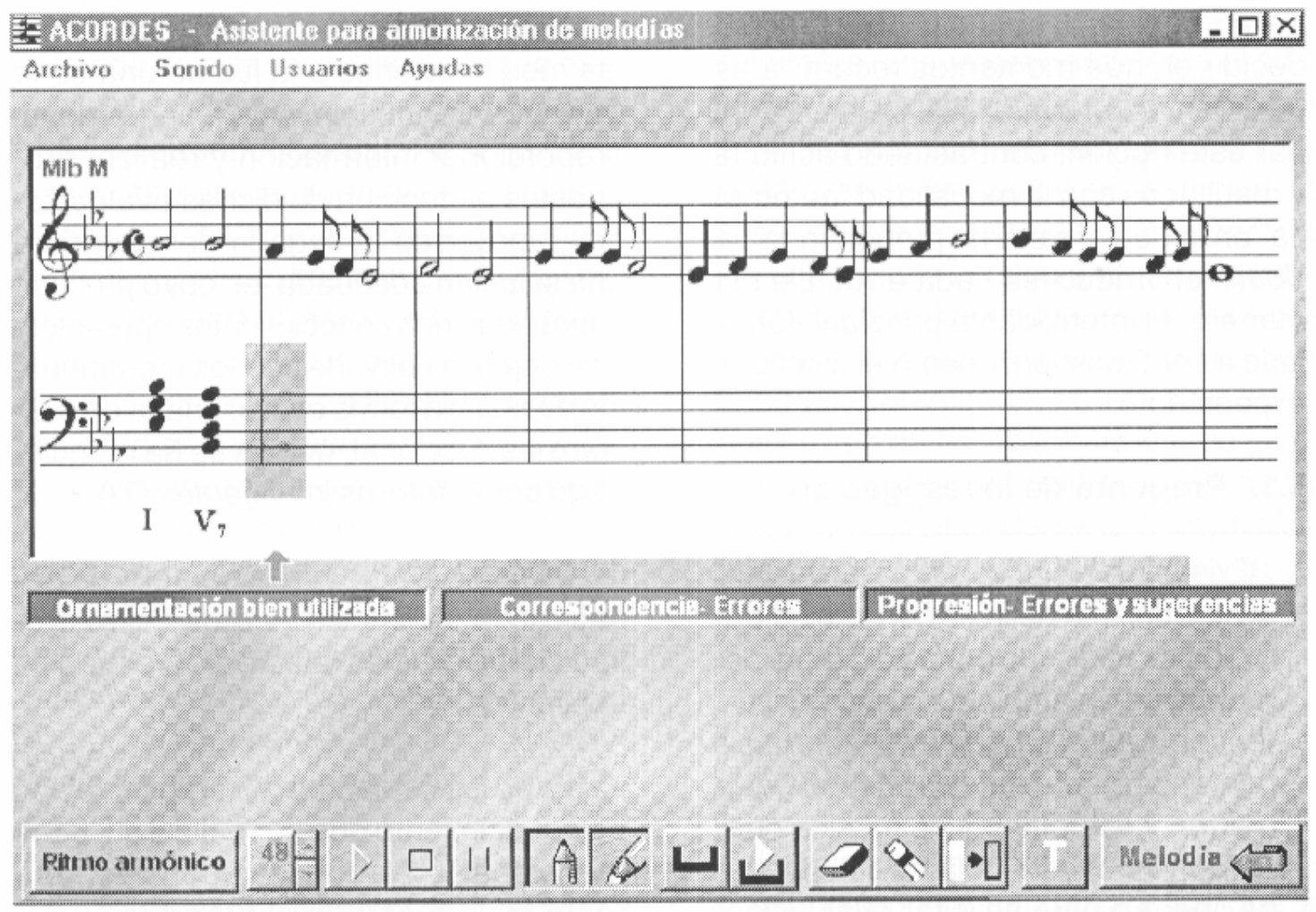

Figura 2. Página de armonización de la aplicación acordes

En el citado artículo se aplica el concepto de alfabeto, denominando así a un conjunto ordenado de alturas-clase o cromas. Una altura-clase incluye, por ejemplo, todos los "LA" sin tener en cuenta la 8va. en que están, o su altura absoluta.

En la aplicación que se desarrolló dicho alfabeto está constituido por las 12 alturasclase de la escala cromática:

DO, DO\#, RE, RE\#, MI, FA, FA\#, SOL, SOL\#, LA, LA\#, SI.

Con relación a ella, se consideran dos estructuras: la escala Mayor natural, con los elementos 1,3,5,6,8,10,12 del alfabeto, y la escala menor natural, con los elementos $1,3,4,6,8,9,11$ del mismo alfabeto.

Por ejemplo, para el análisis de los acordes tocados, se establece un punto de partida en la escala cromática, como distancia en semitonos a una fónica o nota inicial. Este punto de partida queda determinado por la escogencia de la tonalidad. Las notas del acorde tocado se consideran instancias particulares de las alturas tipo (Kostka, "pitch class cells", ver Kostka S., 89). Es decir, cualquier LA que haya tocado el usuario, es un LA, independientemente de la $8 v a$. en que se encuentre. 


\subsection{Selección enarmónica de alteraciones accidentales (notas alteradas no pertenecientes a la escala)}

\section{Criterio del mejor intervalo}

Entre los aspectos más sencillos de la teoría de la Representación general de la altura de los intervalos (Cambouropoulos, 96), se encuentra la valoración del "mejor intervalo" entre dos posibles del mismo tamaño, que contengan escrituras enarmónicas del primer sonido. Por ejemplo, se decidirá entre la conveniencia de escribir, a partir de la nota alterada, SOL \# - MI o LA b - MI.

Como criterio coadyuvante en la decisión, Cambouropoulos establece la preferencia habitual en las partituras, por elegir el mejor intervalo desde de la nota alterada, en vez de hacerlo hacia la misma.

La figura 3demuestra el funcionamiento del programa Acordes de acuerdo dicho criterio de selección de las notas alteradas:

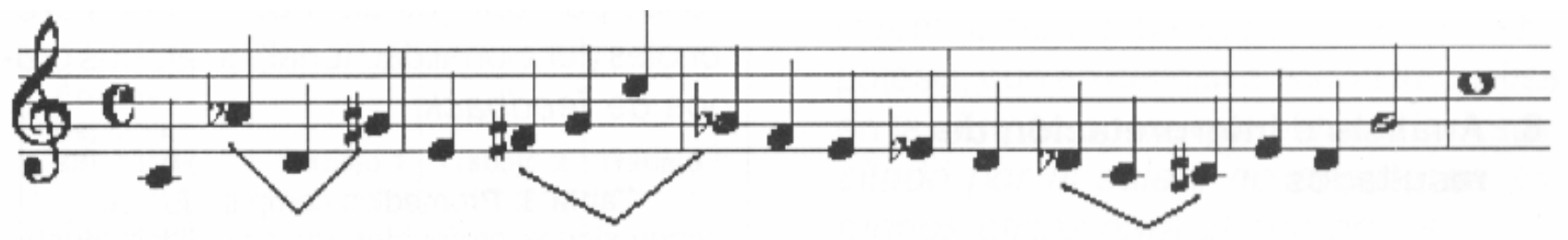

Figura 3. Diferentes escrituras escogidas por el programa para sonidos iguales, según la dirección de la melodía.

La diferencia entre el software al cual dicho autor aplica esta teoría, y el que se está describiendo, radica en para aquél se implementó la capacidad de reconocer la tonalidad del material musical ingresado simplemente como información MIDI, yen nuestro programa se debe especificar la tonalidad de cada melodía nueva que se va a escribir, lo cual reduce el alcance de la tarea.

\section{Metodología}

Para el experimento, se conformaron dos muestras, con estudiantes de nivel básico y universitarios del Departamento de Música de la Universidad Nacional de Colombia. Sus integrantes trabajaron, respectivamente, con el software en modalidad de feedback espontáneo y de feedback solicitado, para armonizar tres melodías de contenido progresivamente más avanzado. El programa registró automáticamente la actividad de cada sujeto, estableciendo indicadores numéricos de los errores, intentos, acordes definitivos, audiciones totales y parciales, consultas y navegaciones en los textos de información.

La pregunta de investigación se subdividió en seis interrogantes, que a su vez permitieron establecer las variables del experimento. En general, estos 6 indicadores permitieron interpretar, mediante pruebas estadísticas de significación, los resultados de la comparación entre ambos tipos de feedback, para estas variables

1. Cantidad de intentos por cada trabajo (índice de eficiencia).

2. Volumen de consultas. 
3. Funcionalidad tonal.

4. Relación entre errores comunicados y búsqueda de información (índice de aprovechamiento del feedback).

5. Calidad de resultados finales.

6. Balance de modalidades cognitivas.

\section{Análisis e interpretación de resultados}

Los resultados de las pruebas indicaron:

8.1. Escasa o nula significación para las diferencias entre índices de eficiencia (inversos de la proporción de intentos fallidos) en la armonización de las dos melodías iniciales, y mayor eficiencia, con ligera ventaja en la tercera melodía, a favor del grupo de feedback solicitado.

Tabla 1. Comparación de índices de eficiencia entre ambos grupos, para cada trabajo.

\begin{tabular}{|l|c|c|c|}
\hline $\begin{array}{l}\text { Intentos/ } \\
\text { Acordes } \\
\text { puestos }\end{array}$ & Trabajo 1 & Trabajo 2 & Trabajo 3 \\
\hline $\begin{array}{l}\text { Feedback } \\
\text { Espontáneo }\end{array}$ & 4.1 & 4 & 3.3 \\
\hline $\begin{array}{l}\text { Feedback } \\
\text { Solicitado }\end{array}$ & 3.7 & 3 & 2.8 \\
\hline
\end{tabular}

8.2. Disminución significativa del volumen de consultas entre la ira. y la última melodía en el grupo de feedback solicitado (tabla 2).

Tabla 2. Promedios de consultas para los 3 trabajos en ambos grupos.

\begin{tabular}{|l|c|c|c|}
\hline $\begin{array}{l}\text { Total } \\
\text { Consultas+ } \\
\text { Navegantes }\end{array}$ & Trabajo 1 & Trabajo 2 & Trabajo 3 \\
\hline $\begin{array}{l}\text { Feedback } \\
\text { Espontáneo }\end{array}$ & 4 & 2.9 & 3 \\
\hline $\begin{array}{l}\text { Feedback } \\
\text { Solicitado }\end{array}$ & 3 & 2.9 & 1.6 \\
\hline
\end{tabular}

8.3. Diferencias no significativas entre los porcentajes de progresiones preferibles (funcionalidad tonal) de ambos grupos de feedback.

Tabla 3. Promedios comparados de progresiones preferibles (funcionalidad tonal).

\begin{tabular}{|l|c|c|c|}
\hline $\begin{array}{l}\text { \% de } \\
\text { Progresiones } \\
\text { preferibles }\end{array}$ & Trabajo 1 & Trabajo 2 & Trabajo 3 \\
\hline $\begin{array}{l}\text { Feedback } \\
\text { Espontáneo }\end{array}$ & 38.7 & 32.2 & 49 \\
\hline $\begin{array}{l}\text { Feedback } \\
\text { Solicitado }\end{array}$ & 40.7 & 30.7 & 44.8 \\
\hline
\end{tabular}


8.4. Se intentó establecer el grado de correlación entre los avisos de error y las consultas informativas, como un indicador del aprovechamiento del feedback para la obtención de información. El grupo de feedback solicitado dio una correlación alta y creciente a lo largo de las tres melodías, y siempre mayor, para cada una de ellas, que el grupo de feedback espontáneo. La figura 4 muestra el caso de la melodía 3.

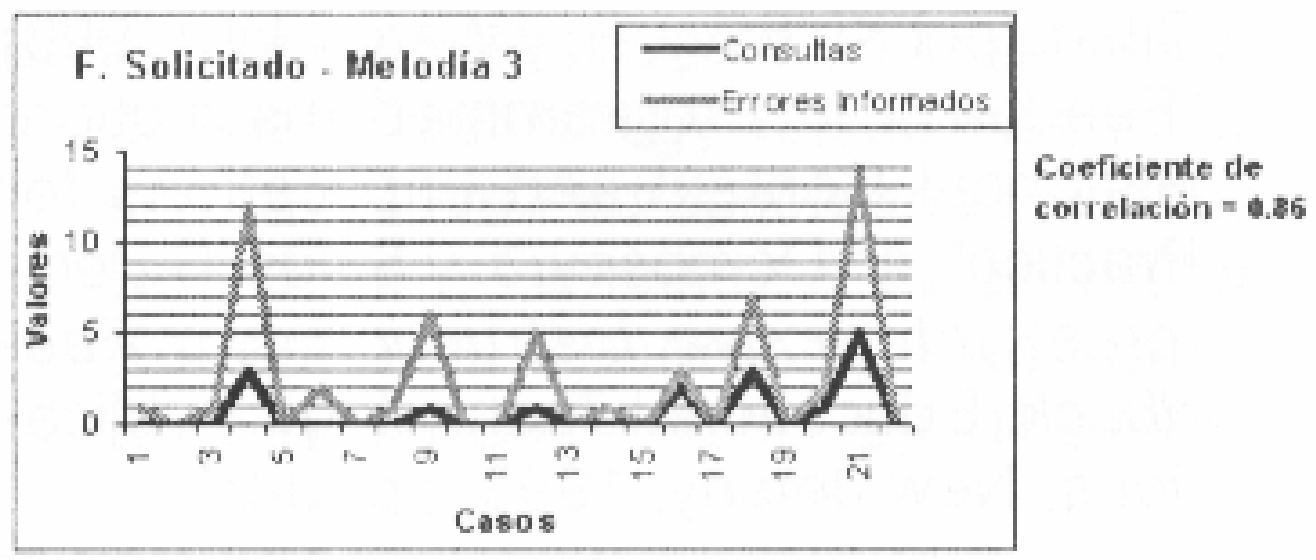

Figura 4. Correlación entre avisos de error y consultas informativas, con feedback solicitado.

8.5. Al terminar cada trabajo, muchos sujetos dejan sin resolver algunos problemas señalados por el software. Las diferencias entre ambos grupos de feedback no resultaron significativas.

Tabla 4. Porcentajes de errores dejados sin corregir.

\begin{tabular}{|l|c|c|c|}
\hline $\begin{array}{l}\text { Errores } \\
\text { Dejados } \\
\text { Sin corregir }\end{array}$ & Trabajo 1 & Trabajo 2 & Trabajo 3 \\
\hline $\begin{array}{l}\text { Feedback } \\
\text { Espontáneo }\end{array}$ & 1.5 & 0.4 & 0.6 \\
\hline $\begin{array}{l}\text { Feedback } \\
\text { Solicitado }\end{array}$ & 1.7 & 1.1 & 0.9 \\
\hline
\end{tabular}

8.6. Se intentó observar la relación entre dos aspectos del proceso de conocimiento musical: la vía informativa-teórica (mediante textos y ejemplos), y una aproximación más sensible al objeto - los acordes-, consistente en la apreciación auditiva de resultados totales o parciales. Se pudo establecer una diferencia importante en este comportamiento cognitivo, entre dos situaciones: la de haber recibido un informe de error, o la de no haber recibido dicho informe, en la acción precedente a la audición o la consulta. En efecto, las consultas al Libro de ayudas no provocadas por avisos de error fueron siempre menores que las audiciones, y en cambio, las veces en que el usuario prefirió escuchar después de enterarse de un error, fueron notablemente inferiores a las consultas realizadas.

Adicionalmente, se aplicó observación cualitativa, la cual permitió obtener interesantes sugerencias de los sujetos con respecto a posibles mejoras del software y otros aspectos.

En general, las pruebas estadísticas realizadas arrojan muy pocas diferencias significativas entre ambos tipos de feedback. Si bien esto no implica una confirmación de 
que, a nivel de procesos cognitivos, operen con eficacia similar, estos resultados no obligan a descartar esta semejanza. De hecho, una próxima investigación debería partir de dicha hipótesis, que en definitiva podría traducirse en a afirmación de que el feedback solicita do por el sujeto no demuestra ser menos eficaz para el aprendizaje de la armonización de melodías, que el feedback ofrecido espontáneamente por el software ante la producción de errores o situaciones a típicas.

Es posible que la heterogeneidad del comportamiento de los sujetos en ambas muestras -observable en los valores altos de las varianzas - haya contribuido a restar significación a ciertas tendencias divergentes, de todas maneras constatadas.

\section{Conclusiones}

Las pruebas estadísticas no arrojan diferencias significativas entre ambos tipos de feedback para la mayoría de los ítems mencionados más arriba, pero la dispersión de datos presentada en las muestras podría influir en estos resultados. Sin embargo, hay una tendencia a un mejor aprovechamiento del feedback solicitado (genera más consultas que el espontáneo) cuando se produce un aviso de error.

La investigación sugiere la conveniencia de combinar ambos tipos de feedback en software del estilo del que se ha descrito, de modo que el usuario pueda migrar de una modalidad a otra voluntariamente. Sin embargo, alguna claridad conceptual debe alcanzarse para un mejor aprovechamiento del feedback solicitado, mientras que el feedback espontáneo advierte a tiempo los errores elementales de estudiantes de poca experiencia, permitiéndoles no acumular situaciones problemáticas cuya solución, una vez avanzado el trabajo, presentaría excesivas dificultades.

En definitiva, la tecnología de la información provee recursos óptimos para el desarrollo de materiales educativos dotados de niveles de inteligencia artificial, adecuados para el aprendizaje de la armonización de melodías, mediante el apoyo de una combinación de diversas formas de feedback adaptables a los distintas momentos y particularidades del proceso cognitivo de cada estudiante.

\section{Bibliografía}

Berz W. y Bowman J. Applications of Research in Music Technology. Muslo Educafors National Conference, Resten, VA, EE. UU., 1994.

Cambouropoulos; E. "A General Pitch Interval Represenfations: Theory and Applications, Journal of New Muslo Research, vol. 25,1996, pp. 231-251,

Crowder, N.A. The arithmetic of comp uters. Santa Barbara, CA: Western Design. Citado por Kulhavy, R. y Wager, W. (1993) "Feedback in Programmed Instruction: Historical Context and Implications for Practice", en Dempsey, J. y Sales, G. (editores) Interactive Instruction and Feodback. Educational Technology Publications, New Jersey, 1993, pp. 3-20.

Crowder, N.A. "Intrinsic and extrinsic programming, en J.E. Coulson (Ed.), Programmed learning and computerbased instruction. New York: John Wiley \& Sons, 58- 66. Citado por Kulhavy, R. y Wager, W. (1993) "Feedback in Programmed Instruction: Hisforical 
Confexf and Implications for Practice', en Dempsey, J. y Sales, O. (editores, 1993) Interactive ínstruction and Feedback. New Yersey, Educational Technology Publications, 1961, pp. 3-20.

Escudero Muñoz, J.M. Modelos didácticos. Barcelona, Oikos-tau, cap. 2,1981, pp. 47-92.

Kostka, Stefan. Materials and Techniques of Twentieth-Century Music. New Jersey, Prentice Hall, 1989.

Kulhavy, R. W. y Stock, W. A. Feedback in written instruction: The place of response certitude. Educational Psychology Review, 1, 279-308. Citado por ROSS, Steven y Morrison, Gary. "Using feedback to adapt Instruction for Individuals", en Interactive Instruction and Feedback. Dempsey \& Sales, Editors. Educational Tecnology Publication. Englewood Cliffs, N. Jersey, 1993, 1989, pp 177-195.

Kulhavy, R. y Wager, W. "Feedback in Programmed instruction: Historical Confext and Implications ter Practice", en Dempsey, J. y Sales, O. (editores, 1993) Interactive Instruction and Feedback. New Jersey, Educational Technology Publicafions, 1993, pp. 3-20.

Ross, Steven y Morrison, Gary. "Using feedback te adapt Instruction ter Individuals", en Interactíve Instruction and Feedback. New Jersey, Dempsey \& Sales, Editors. Educational Tecnology Publication. Englewood Cliffs, 1993, pp 177-195.

Sales, Gregory. "Adapted and Adaptive Feedback in Technology-Based Instruction" en interactive Instruction and Feedback. New Jersey, Dempsey \& Sales, Editors. Educational Tecnology Publication. Englewood Cliffs, 1993, pp. 159-175.

Schaffer, John W. Microcomputer-Based Intelligent Tutoring Systems: An Assessment. ERIC Reproduction Document Service. Office of Educational Rescarch and Improvement, U.S. Dept. of Education. Ficha ED 307 196, 1988.

Wager, W., yWager, 8. Presenting questions, processing responses, and providing feedback in CAI. Journal of Instructional Development, 8(4), 2-8. Citado por SALES, Gregory. Adapted and Adaptive Feedback in Technology-Based Instruction. En Interactive Instruction and Feedback. Dempsey \& Sales, Editors. Educational Tecnology Publication. Englewood Cliffs, N. Jersey, 1993, pp. 159-175.

West R., Howell P., Cross, I. "Modelling Perc (1986). eived Musical Structure, en Howell, P.; Cross, I.; West, R. (editores) Musical structure and cognition. London, Academic Press inc, 1985, págs. 21-52. 